\title{
Development of Streptococcus thermophilus lacZ as a reporter gene for Candida albicans
}

\author{
M. Andrew Uhl and Alexander D. Johnson† \\ Author for correspondence: M. Andrew Uhl. Tel: +1 415502 0859. Fax: +1 4154768201. \\ e-mail: uhl@socrates.ucsf.edu
}

Department of Microbiology and Immunology, University of California, San Francisco, CA 94143-0414, USA

\begin{abstract}
The study of gene regulation in many organisms has been facilitated by the development of reporter genes. The authors report the use of lacZ from Streptococcus thermophilus, a gene encoding a $\beta$-galactosidase, as a reporter for the fungal pathogen Candida albicans. As test cases, Strep. thermophilus lacZ was placed under control of three different $C$. albicans promoters: MAL2 (maltase), inducible by maltose; HWP1 (hyphal cell wall protein), induced by conditions that promote filamentous growth; and ACT1 (actin). These constructs were each integrated into the $C$. albicans genome and $\beta$ galactosidase activity was readily detected from these strains, but only under the appropriate growth conditions. $\beta$-Galactosidase activity could be detected by several methods: quantitative liquid assays using permeabilized cells, colorimetric assays of colonies replicated to paper filters, and in situ coloration of colonies growing on medium containing the indicator $X$-Gal. These results show the usefulness of Strep. thermophilus lacZ as a monitor of gene regulation in this medically important yeast.
\end{abstract}

Keywords: $\beta$-galactosidase, yeast, fungal pathogen, gene regulation

\section{INTRODUCTION}

Candida albicans is a commensal yeast of warm-blooded animals and has been recognized as an opportunistic pathogen for many years. C. albicans can assume a variety of morphologies, including budding yeast (blastospores) as well as pseudohyphal and hyphal forms; all of these are found at sites of infection (Odds, 1988). A number of growth conditions can be utilized in vitro to induce the transition between morphological forms of C. albicans. Signals which influence the yeast to hyphal transition include temperature, $\mathrm{pH}$ and the nutrient composition of the growth media (Odds, 1988), and several genes with roles in the morphological transition have been identified (Liu et al., 1994; Braun \& Johnson, 1997; Csank et al., 1997, 1998; Lo et al., 1997; Newport \& Agabian, 1997; Alex et al., 1998; Gale et al., 1998; Timpel et al., 1998; Stoldt et al., 1997; Leberer et al., 1996, 1997; Calera et al., 2000; Brown \& Gow, 1999).

Studies of transcriptional regulation in C. albicans have

† Present address: Department of Biochemistry and Biophysics, University of California, San Francisco, CA, USA. been limited by several properties of the organism; e.g. it is diploid with a little-understood sexual cycle, making conventional genetic analysis impossible (Odds, 1988). Additionally, expression of foreign genes, including reporter genes, in C. albicans has been complicated by the alternative codon usage of the organism. The codon CTG is read as a serine in C. albicans but as a leucine in other organisms (Ohama et al., 1993). Escherichia coli lacZ, which has been successfully adapted as a reporter gene in many organisms, contains 51 CTG codons (Gilbert \& Maxam, 1973); therefore successful expression of a functional enzyme seems unlikely without extensive alteration. Other common reporter genes, such as nidA ( $\beta$-glucuronidase) also contain several CTG codons (Blanco et al., 1985). We were particularly interested in developing a $\beta$-galactosidase as a reporter for C. albicans because of the extensive variety of substrates available for this enzyme. In addition, $C$. albicans is not known to have an endogenous $\beta$ galactosidase and cannot utilize lactose as a carbon source (Kwon-Chung, 1992).

Here we report the use of lacZ from Streptococcus thermophilus as a versatile reporter gene for C. albicans. We show that $\beta$-galactosidase activity can be readily detected by a variety of methods when lac $Z$ is expressed from several C. albicans promoters. 


\section{METHODS}

Strains and plasmids. pRH116 was obtained from Larry McKay (Dept of Food Science and Nutrition, University of Minnesota, St Paul) and contains Strep. thermophilus lacZ in a pBR322-based vector (Schroeder et al., 1991). The vectors utilized for LacZ expression were constructed as follows. pAU13 contains the URA3 gene from C. albicans inserted into $\mathrm{XbaI} /$ Ecl136II-digested pBluescript KS + (Stratagene) as an XbaI-Scal fragment from pDBV52 (Brown et al., 1996). pAU15, the MAL2 expression vector, was created by amplifying a $550 \mathrm{bp}$ region upstream of the MAL2 coding sequence with the primers MAL2-5' (GAAGGTACCAGATTGATATTTTTGTCTAGTACC) and MAL2p3' (TCTCACTCGAGATGTAGTTGATTATTAGTTAAAC) using C. albicans CAI4 genomic DNA. A 250 bp downstream region of MAL2 was amplified from C. albicans CAI4 genomic DNA using the primers UTR5' (CCTGGGATCCTAAATATACACTAGATGCTATC) and MAL2-3' (CCTGTCTAGAAGACATACGCTTTGCAGGTGGTGTT). The MAL2 upstream and downstream fragments were cloned into pAU13 to create pAU15, which is a MAL2 expression vector containing a XhoI-SalI-HindIII-SmaI-BamHI polylinker following the MAL2 promoter. The HWP1 expression vector was created in a similar fashion, by amplifying an upstream region of $H W P 1$ with the primers HWP1-P5' (GTCCTGGTACCAAAAACAAGGAATTCGGAAATTC) and HWP1-P3' (CTCCTTCTCGAGATTGACGAAACTAAAAGCGAGTG). The downstream region of $H W P 1$ was amplified with the primers HWP1-U5' (GAAGAGGATCCTTCTAATAACTGATACTAAGTTTT) and HWP1-U3' (CTCTTTCTAGAATTGTAAAAAAAAGGGGTATTTATT). The resulting fragments were cloned into pAU13, resulting in pAU64, which contains a HindIII-EcoRV-PstI-SmaI polylinker following the HWP1 promoter. The ACT1 expression vector was created by amplifying a $1 \cdot 1 \mathrm{~kb}$ region upstream of ACT1 from C. albicans CAI4 genomic DNA with the primers ACT1-P5' (CTCTCGGTACCAGAGCTATTAAGATCACCAGCCT) and ACT1-P3' (CTCTTCCTCGAGTTTGAATGATTATATTTTTTTAATATTAA). The ACT1 downstream region was amplified with the primers ACT1-U5' (GAAGAGGATCCGAGTGAAATTCTGGAAATCTGGAA) and ACT1U3' (CTCTTTCTAGAATTTTATGATGGAATGAATGGGATG). These fragments were cloned into pAU13 to create pAU34, which contains an XhoI-ClaI-HindIII-EcoRVPstI-SmaI-BamHI polylinker following the ACT1 promoter region.

The Strep. thermophilus lacZ gene was amplified from pRH116 using the primers LacZ5' (GTGTCCCGGGTCCATGAACATGACTGAAAAAATTCAA) and LacZ3' (TCCACGAGGATCCCTAATTTAGTGGTTCAATCATGA$A G)$ and cloned into the various expression constructs to create the following plasmids: MAL2-lacZ (pAU22), HWP1lacZ (pAU95) and ACT1-lacZ (pAU36). Alteration of the CTG codon to TTA in lacZ was done by PCR-based mutagenesis using the primer pairs LacZ-1631 (ATGGTGTCGTGAGTTTGA) and LacZm3' (CAGTTGCTTCTCTTAAGACACAAGCAACTTCGTAAATTTG), and LacZm5' (GTTGCTTGTGTCTTAAGAGAAGCAACTGAATGGGCTCC) and LacZ3'. The resulting PCR fragments were mixed and served as a template for amplification with the primers LacZ-1631 and LacZ3'; this was cloned into pAU22 to give pAU106. Presence of the mutation was screened for by creation of an AflII site and confirmed by sequencing. For expression of Strep. thermophilus lacZ in Saccharomyces cerevisiae, lacZ was amplified with Pfu DNA polymerase (Stratagene) using linearized pAU22 as a template and the primers LacZ5' and MAL2-3'Bln (CCTGCCTAGGAGACATACGCTTTGCAGGTGGTGTT). The resulting product was digested with $X m a \mathrm{I}$ and $B \ln \mathrm{I}$ and cloned into XmaI/ XbaI-cut pDK20 (a gift from Doug Kellogg, Sinsheimer Laboratories, Dept of Biology, University of California, Santa Cruz).

C. albicans methods. C. albicans strain CAI4 (ura3:: imm434/ ura3::imm434) served as the parent strain for all manipulations (Fonzi \& Irwin, 1993). Linearized DNA was transformed into CAI4 by the modified lithium acetate method (Hill et al., 1991; Gietz et al., 1995), and transformants were selected on medium lacking uridine (Guthrie \& Fink, 1991). Standard recipes were utilized for all media, and carbon sources such as maltose, glucose, galactose or lactose were added at a final concentration of $2 \%(\mathrm{v} / \mathrm{v})$ (Guthrie \& Fink, 1991). For expression of lacZ in Sacch. cerevisiae, strain W303 (ade2-1 trp1-1 cau1-100 leu2-3,112 his3-11 ura3 psi+) was utilized (Guthrie \& Fink, 1991). Plasmids were introduced into W303 by lithium acetate transformation (Hill et al., 1991; Gietz et al., 1995).

$\boldsymbol{\beta}$-Galactosidase assays. C. albicans $\beta$-galactosidase assays were performed as described by Ausubel et al. (1992) for Sacch. cerevisiae with minor modifications. For liquid assays, $1 \mathrm{ml}$ cells was resuspended in an equal volume of $\mathrm{Z}$ buffer (Ausubel et al., 1992) and placed on ice. The $\mathrm{OD}_{600}$ was determined for each sample. Then 10-100 $\mu$ l of cells was added to $Z$ buffer to a final volume of $1 \mathrm{ml}$, and the cells were permeabilized with $15 \mu \mathrm{l} 0 \cdot 1 \%$ SDS and $30 \mu \mathrm{l}$ chloroform. There was no appreciable change in activity when alternative methods such as toluene or glass beads were used for permeabilizing cells. Cells were equilibrated at $37^{\circ} \mathrm{C}$ for $5 \mathrm{~min}$, then $0.2 \mathrm{ml} \mathrm{ONPG}\left(4 \mathrm{mg} \mathrm{ml}^{-1}\right)$ was added and the cells were mixed and incubated at $37^{\circ} \mathrm{C}$. Reactions were stopped by addition of $0.5 \mathrm{ml} 1 \mathrm{M} \mathrm{Na}_{2} \mathrm{CO}_{3}$, spun for $5 \mathrm{~min}$ at $10000 \mathrm{~g}$ and the $A_{420}$ and $A_{550}$ were read. Units of activity were determined by the standard equation given by Ausubel et al. (1992).

Filter assays were performed as described by Ausubel et al. (1992). Patched colonies of C. albicans were replica-plated onto solid medium with an overlay of a circular Whatman filter and grown overnight. Filters were frozen in liquid nitrogen and incubated in $3 \mathrm{ml} \mathrm{Z}$ buffer with $20 \mu \mathrm{l} 3 \% \mathrm{X}-\mathrm{Gal}$ and incubated at $37^{\circ} \mathrm{C}$.

Visual screens for C. albicans were carried out by patching colonies onto X-Gal plates (Ausubel et al., 1992). The standard formulation for X-Gal plates was utilized for most applications. A slightly modified recipe proved more sensitive for detection of $\beta$-galactosidase, although strains grew more slowly on this medium (X-Gal Modified Medium, XMM). XMM contained $1.7 \mathrm{~g}$ Yeast Nitrogen Base (without amino acids or ammonium sulfate), $20 \mathrm{~g}$ glucose, $5 \mathrm{~g}$ ammonium sulfate and $20 \mathrm{~g}$ agar in $930 \mathrm{ml} \mathrm{H_{2 }} \mathrm{O}$. After autoclaving, $70 \mathrm{ml}$ $1 \mathrm{M}$ potassium phosphate $\mathrm{pH} 7 \cdot 0$ and $2 \mathrm{ml}$ of a $20 \mathrm{mg} \mathrm{ml}^{-1} \mathrm{X}$ Gal solution were added.

\section{RESULTS AND DISCUSSION}

\section{Assays of $\beta$-galactosidases in C. albicans}

We reasoned that $\beta$-galactosidases from organisms with high percentages of adenosine/thymine genomic content might be biased against CTG codons; a search against various databases revealed two $\beta$-galactosidases that contained relatively few CTG codons. LAC4 from 


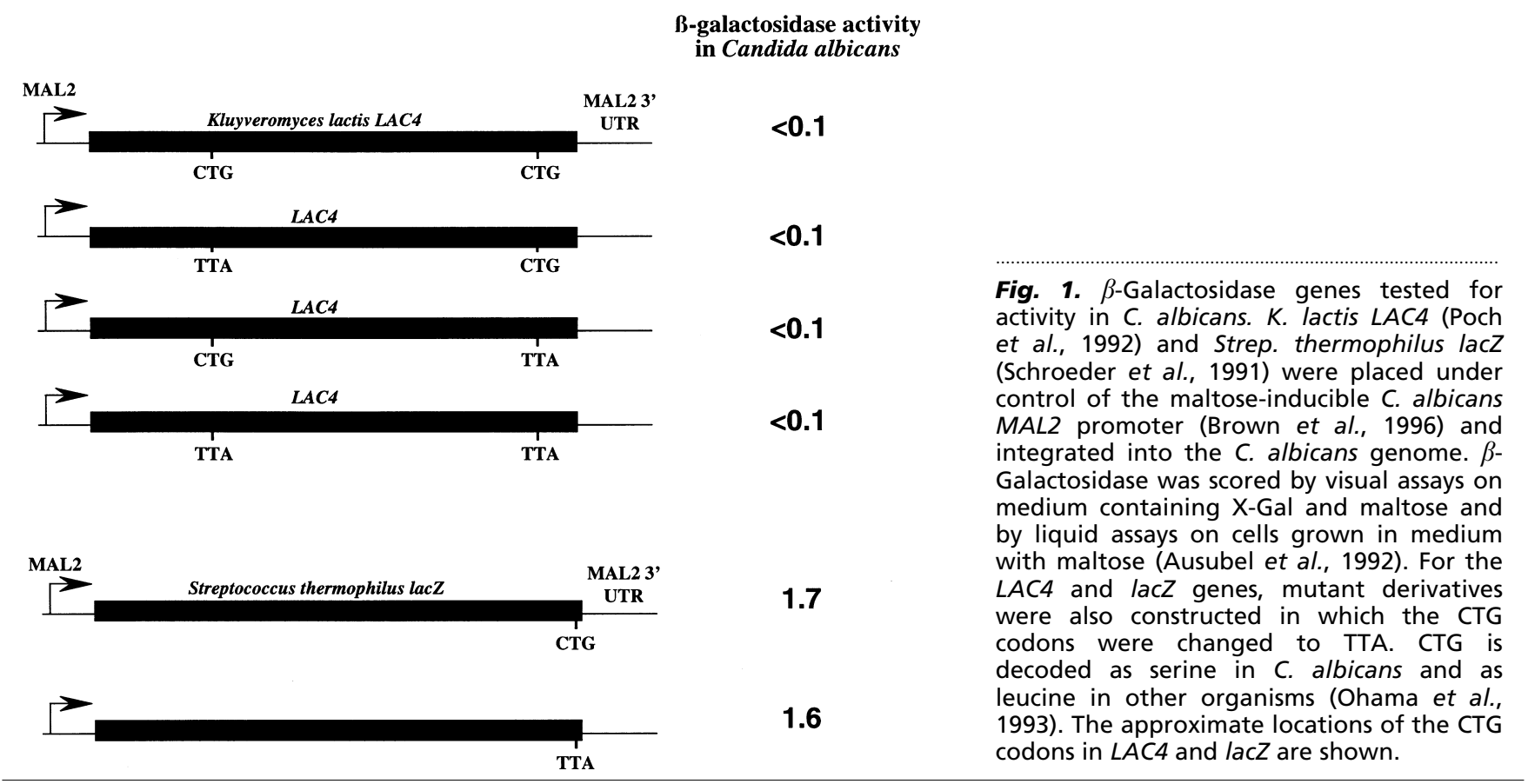

the yeast Kluyveromyces lactis contains only two CTG codons, while lacZ from the thermophilic bacterium Strep. thermophilus has only one CTG codon (Schroeder et al., 1991; Poch et al., 1992). The single leucine encoded by CTG in the Strep. thermophilus lacZ is at the extreme C-terminus of the LacZ protein in a nonconserved region of $\beta$-galactosidases, while one of the leucines encoded by a CTG codon in the K. lactis LAC4 gene is in a position conserved as a hydrophobic residue in $\beta$-galactosidases (Jacobson et al., 1994) (Fig. 1). Both CTG codons in LAC4 were changed to TTA in order to specify leucines at these positions. Either wild-type $L A C 4$ or the mutant derivatives were integrated into the C. albicans genome and expressed from the MAL2 (maltase) promoter. MAL2 is induced by maltose and repressed by glucose (Brown et al., 1996). No activity was detected in strains with the MAL2-LAC4 wild-type and MAL2-LAC4 mutant derivatives under inducing conditions using both liquid assays and qualitative plate assays (Fig. 1). Although LAC4 has previously been described as a possible reporter gene in C. albicans (Leuker et al., 1992), its usefulness in our hands appeared limited. When LAC4 was expressed from the ACT1 promoter, $\beta$-galactosidase activity could only be detected in a limited number of transformants; these presumably represented strains with multiple integration events (Leuker et al., 1992).

When Strep. thermophilus lacZ (unaltered in its CTG codon) or lacZ with the single CTG codon altered to TTA was expressed from the MAL2 promoter in C. albicans, $\beta$-galactosidase activity could be readily detected by several means (Fig. 1). Colonies of $C$. albicans expressing MAL2-lacZ turned blue on solid medium containing both maltose and the chromogenic substrate X-Gal after a few days' growth. No such activity was detected when colonies were grown on the same medium containing glucose instead of maltose. Maltose-dependent activity was also detected by liquid assays (see below and Table 1).

\section{Expression of lacZ from a variety of promoters}

Since lacZ expression could be detected from the MAL2 promoter, we examined its use for monitoring promoter activity from two other C. albicans genes, ACT1 and HWP1 (Fig. 2). The promoter for the ACT1 (actin) gene has previously been used for expression of foreign genes in C. albicans (Leuker et al., 1992). ACT1 promoter expression is constant under many conditions but has been shown to be regulated by starvation and growth phase in C. albicans (Delbruck \& Ernst, 1993). HWP1 expression is minimal when C. albicans is growing in the budding yeast form and highly induced during conditions that promote filamentous growth (Staab et al., 1996). Quantitative liquid assays were performed on C. albicans strains expressing MAL2-lacZ, ACT1-lacZ or HWP1-lacZ from constructs that had been integrated in the genome (Table 1). Strains were grown at $30^{\circ} \mathrm{C}$ in YEP-glucose or YEP-maltose (budding yeast morphology) or at $37^{\circ} \mathrm{C}$ in YEP-glucose with $10 \%$ fetal calf serum (hyphal/pseudohyphal morphology).

In the MAL2-lacZ strains, $\beta$-galactosidase activity was significantly induced ( $>20$-fold) by growth in maltose compared to growth in glucose; activity was detected at only background levels in glucose (Table 1), where background levels are defined as the very low levels of $\beta$ galactosidase activity observed in the absence of the Strep. thermophilus lacZ reporter gene. Expression of 


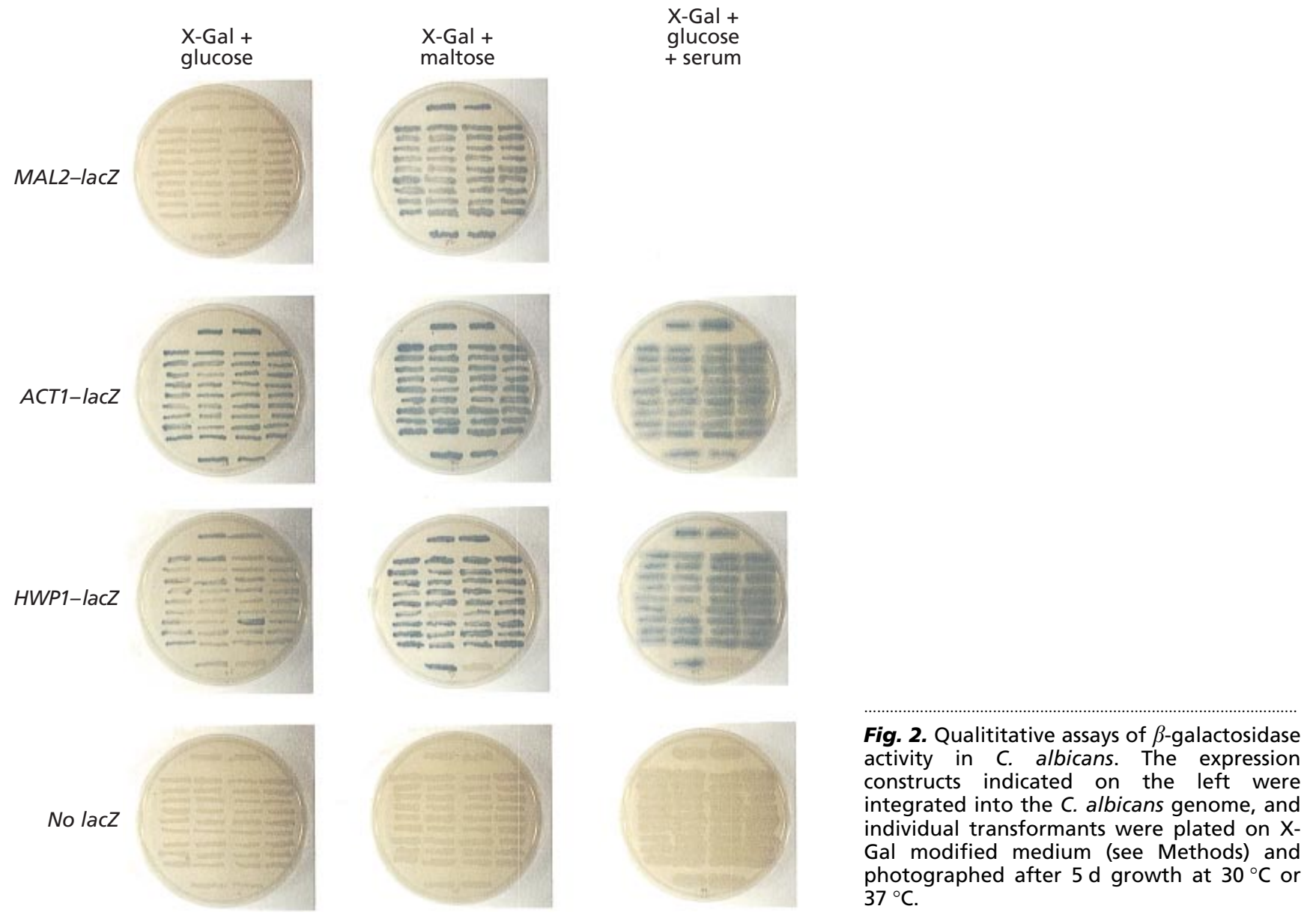

Table 1. Expression of lacZ from various promoters in C. albicans

Cultures were grown in YEP + glucose (Glc), YEP + maltose $(\mathrm{Mal})$ or YEP + glucose $+10 \%$ fetal calf serum. Cells were permeabilized and $\beta$-galactosidase assays were performed in $Z$ buffer using ONPG as a substrate (Ausubel et al., 1992). Values represent the mean of three independent transformants; units are expressed according to the standard protocol for Sacch. cerevisiae (Ausubel et al., 1992). MAL2-lacZ* refers to the Strep. thermophilus lacZ gene in which the single CTG codon was changed to TTA.

\begin{tabular}{|lccc|}
\hline Construct & \multicolumn{3}{c|}{$\beta$-Galactosidase activity } \\
\cline { 2 - 4 } & + Mal & + Glc & + Glc + serum \\
& & & \\
\hline MAL2 & $<0 \cdot 1$ & $<0 \cdot 1$ & $<0 \cdot 1$ \\
MAL2-LacZ & $1 \cdot 7 \pm 0 \cdot 1$ & $<0 \cdot 1$ & $<0 \cdot 1$ \\
MAL2-LacZ* & $1 \cdot 6 \pm 0 \cdot 1$ & $<0 \cdot 1$ & \\
ACT1 & $<0 \cdot 1$ & $<0 \cdot 1$ & $<0 \cdot 1$ \\
ACT1-LacZ & $7 \cdot 0 \pm 0 \cdot 5$ & $9 \cdot 0 \pm 0 \cdot 5$ & $10 \pm 0 \cdot 5$ \\
HWP1 & $<0 \cdot 1$ & $<0 \cdot 1$ & $<0 \cdot 1$ \\
HWP1-LacZ & $1 \cdot 1 \pm 0 \cdot 1$ & $<0 \cdot 1$ & $24 \pm 4$ \\
SC5314 (WT) & $<0 \cdot 1$ & $<0 \cdot 1$ & $<0.1$ \\
\hline
\end{tabular}

MAL2-lacZ was not induced significantly under conditions that promoted filamentous growth (Table 1). We noticed that approximately $10 \%$ of strains transformed with our lacZ constructs expressed significantly higher levels of $\beta$-galactosidase than did the majority; these strains were assumed to represent multiple integrants and were not studied further. Examples of this are shown in Fig. 2. Additionally, approximately 5\% of strains did not express detectable levels of $\beta$ galactosidase; these presumably resulted from integration into the genome at sites other than the intended locus or from rearrangements that occurred subsequent to transformation.

Expression of the ACT1-lacZ construct was constant during all growth conditions, varying less than $1 \cdot 5$-fold during all growth conditions (Table 1). The ACT1 gene is known to be downregulated by starvation and during growth-phase changes; these conditions were not tested for the ACT1-lacZ fusion (Delbruck \& Ernst, 1993; Swoboda et al., 1994). In contrast, the HWP1-lacZ construct was strongly induced by growth under filamentous conditions, including YEP-glucose $+10 \%$ fetal calf serum at $37^{\circ} \mathrm{C}$. Compared to growth in YEPglucose, the HWP1-lacZ construct was induced approxi- 
mately 250 -fold by growth in YEP-glucose $+10 \%$ fetal calf serum. This is consistent with measurements of RNA levels of HWP1 in cells grown in YEP-glucose or YEP-glucose $+10 \%$ fetal calf serum at $37^{\circ} \mathrm{C}$ (data not shown). HWP1-lacZ was also induced to a lesser degree by substitution of maltose for glucose in the growth medium (see Table 1 and Fig. 2), and utilization of maltose as a carbon source has been reported to be an inducer of filamentous growth in C. albicans (Odds, 1988).

In order to optimize enzymic assays for the Strep. thermophilus $\beta$-galactosidase, we performed assays at various temperatures and $\mathrm{pH}$ values. The temperature optimum of Strep. thermophilus LacZ was found to lie between 45 and $50{ }^{\circ} \mathrm{C}$; enzymic activity fell sharply at temperatures higher than $50^{\circ} \mathrm{C}$. Activity of the enzyme was $2 \cdot 2$-fold higher at $45^{\circ} \mathrm{C}$ than at $30^{\circ} \mathrm{C}$. The $\mathrm{pH}$ optimum of the enzyme was between $6 \cdot 8$ and $7 \cdot 2$.

\section{Expression of lacZ does not allow growth on lactose as a sole carbon source}

Since expression of ACT1-lacZ was independent of carbon source, this allowed a test of whether C. albicans expressing $\beta$-galactosidase could utilize lactose as a sole carbon source. We grew C. albicans SC5314 (wild-type) and C. albicans CA361 (ACT1::lacZ) on minimal medium with glucose (SG), minimal medium with lactose (SL) and minimal medium without any carbon source (S). No growth of SC5314 or CA361 was observed on SL or S medium, while both strains grew on SG medium (data not shown). A similar phenotype has been noticed for Sacch. cerevisiae strains expressing $\beta$ galactosidase, and expression of a lactose permease was necessary before lactose could be utilized as a carbon source (Sreekrishna \& Dickson, 1985).

\section{Qualitative assays of $\beta$-galactosidase activity}

Growth of cells on medium containing X-Gal provides a useful method of screening large numbers of cells for the expression of $\beta$-galactosidase activity under particular sets of conditions. When cells were plated on the appropriate medium containing X-Gal, activity was easily detectable from $C$. albicans strains expressing MAL2-lacZ (CAU221), HWP1-lacZ (CAU951) and ACT1-lacZ (CAU361) (Fig. 2). After $2 \mathrm{~d}$, colonies expressing the HWP1-lacZ fusions turned blue on X$\mathrm{Gal}+10 \%$ fetal calf serum, but remained white on X$\mathrm{Gal}+$ glucose or X-Gal+maltose (not shown). However, after $5 \mathrm{~d}$, strains with the HWP1-lacZ fusion began to turn blue on all media (Fig. 2). This result was expected since $H W P 1$ expression is highly induced under a variety of conditions that promote filamentous growth, including starvation, a condition that arises in colonies upon prolonged growth (see below). Colonies of the ACT1-lacZ fusion strain turned visibly blue on all media as soon as $2 \mathrm{~d}$, and developed further after $5 \mathrm{~d}$ (Fig. 3). Colonies of the MAL2-lacZ fusion strain turned blue on X-Gal + maltose medium in $4-5 \mathrm{~d}$, but

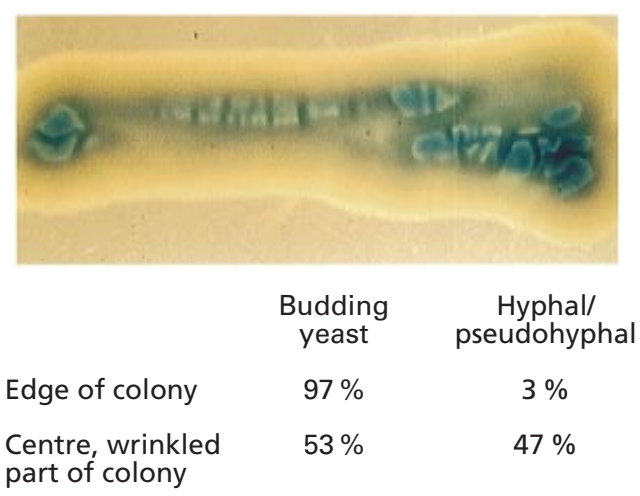

Fig. 3. Expression of the HWP1-lacZ construct in particular regions of a streak of $C$. albicans. A $C$. albicans strain with the integrated HWP1-lacZ construct was grown on X-Gal + maltose at $30^{\circ} \mathrm{C}$ for $3 \mathrm{~d}$. $\beta$-Galactosidase activity is noticeable in regions of the streak that appear wrinkled and in the centre of the streak; these regions contain a higher percentage of filamentous cells than the edge of the streak, which contains $C$. albicans primarily in the budding yeast form. Cell morphology was quantified by resuspending sections of colonies in liquid culture and scoring 300 cells for morphology.

remained white on X-Gal+glucose medium (Fig. 2). The longer developing time of the MAL2-lacZ fusion strains compared with the other strains is consistent with the lower level of expression as detected by the liquid assays (Table 1).

In addition to its usefulness in distinguishing expressing from non-expressing colonies, growth on X-Gal medium can also be used to discern changes in gene expression that occur within a colony. Fig. 3 depicts a streak of a $C$. albicans strain containing the HWP1-lacZ fusion that had been grown on X-Gal + maltose for $3 \mathrm{~d}$. Most of the streak is white, consistent with the lack of expression of HWP1 by budding cells. However, in the centre of the streak, particularly in areas of wrinkled appearance, groups of cells have begun to express lacZ. Microscopic analysis revealed that many of the cells taken from the centre of the streak were filamentous while almost all those taken from around the edge were in the budding form. It seems likely that conditions in the centre of the streak, perhaps depletion of nutrients, had induced filamentous growth, which is easily monitored by expression of the HWP1-lacZ fusion. No such patterns of expression were seen in colonies of $C$. albicans expressing the ACT1-lacZ fusion.

Although growth on X-Gal medium is a useful monitor of lac $Z$ expression, it can require several days for the colour to develop. For more rapid detection of $\beta$ galactosidase activity, colonies grown on solid medium can be transferred to paper filters, permeabilized by freezing in liquid nitrogen and incubated with X-Gal (Ausubel et al., 1992). Results with filter assays matched results of the liquid $\beta$-galactosidase assays and plate assays (not shown). Positive reactions were obtained after only a few hours' incubation at $37^{\circ} \mathrm{C}$ for $\mathrm{C}$. 
albicans strains with integrated MAL2-lacZ, HWP1lacZ or ACT1-lacZ fusions.

\section{Strep. thermophilus lacZ in Sacch. cerevisiae}

Since it is often useful to compare regulatory pathways in C. albicans and Sacch. cerevisiae (Gimeno et al., 1992), we tested whether Strep. thermophilus lacZ also functioned in Sacch. cerevisiae. Sacch. cerevisiae strains that contain the Strep. thermophilus lacZ fused to the GAL1,10 promoter and integrated in the genome readily exhibit regulated $\beta$-galactosidase activity as determined by filter assays (Guthrie \& Fink, 1991). When grown on YEP-galactose, $88 \%$ of the transformants produced $\beta$ galactosidase activity within $1 \mathrm{~h}$, while no activity was observed when transformants were grown on YEPglucose. No activity was detected from Sacch. cerevisiae stains containing the GAL1,10 vector alone. Thus, Strep. thermophilus lacZ can be shuttled between C. albicans and Sacch.cerevisiae and used as a reporter gene in both yeasts.

The results presented in this paper show that lacZ from Strep. thermophilus is a useful reporter gene for the human pathogen C. albicans. Other reporter genes have been developed for C. albicans, including the sea pansy luciferase (Srikantha et al., 1996), the K. lactis $\beta$ galactosidase LAC4 (Leuker et al., 1992), the URA3 gene from C. albicans (Myers et al., 1995) and the yeastenhanced green fluorescent protein (yEGFP; Cormack et al., 1997). These reporter genes have been used effectively for studies of transcriptional regulation in $C$. albicans (Wirsching et al., 2000; Srikantha et al., 1996, 1997; Stoldt et al., 1997; Leuker et al., 1997). Strep. thermophilus LacZ combines the advantages of sensitivity, simplicity of qualitative assays, and ease of colorimetric visual screens in growing colonies of $C$. albicans.

E. coli lacZ has proved to be a highly versatile reporter gene in Sacch. cerevisiae and has been used to study many aspects of signal-transduction pathways, gene regulation and other cellular processes (Guarente \& Ptashne, 1981; Rose et al., 1981; Guarente, 1983; Burns et al., 1994). We believe that the Strep. thermophilus lacZ reporter gene can be used for many of these same purposes in C. albicans.

\section{ACKNOWLEDGEMENTS}

We thank Burk Braun and Christina Hull for reagents and protocols, Larry McKay for plasmid pRH116, and members of the Johnson lab for advice. This work was supported by a Damon Runyon-Walter Winchell post-doctoral research grant to M.A.U. and NIH grant GM37049 to A.D. J.

\section{REFERENCES}

Alex, L. A., Korch, C., Selitrennikoff, C. P. \& Simon, M. I. (1998). COS1, a two-component histidine kinase that is involved in hyphal development in the opportunistic pathogen Candida albicans. Proc Natl Acad Sci US A 95, 7069-7073.
Ausubel, F. M., Brent, R., Kingston, R. E., Moore, D. D., Seidman, J. G., Smith, J. A. \& Struhl, K. (1992). Current Protocols in Molecular Biology. New York: Greene Publishing Associates and Wiley-Interscience.

Blanco, C., Ritzenthaler, P. \& Mata-Gilsinger, M. (1985). Nucleotide sequence of a regulatory region of the uidA gene in Escherichia coli K12. Mol Gen Genet 199, 101-105.

Braun, B. R. \& Johnson, A. D. (1997). Control of filament formation in Candida albicans by the transcriptional repressor TUP1. Science 277, 105-109.

Brown, A. J. \& Gow, N. A. (1999). Regulatory networks controlling Candida albicans morphogenesis. Trends Microbiol 7, 333-338.

Brown, D. H., Jr, Slobodkin, I. V. \& Kumamoto, C. A. (1996). Stable transformation and regulated expression of an inducible reporter construct in Candida albicans using restriction enzymemediated integration. Mol Gen Genet 251, 75-80.

Burns, N., Grimwade, B., Ross-Macdonald, P. B., Choi, E. Y., Finberg, K., Roeder, G. S. \& Snyder, M. (1994). Large-scale analysis of gene expression, protein localization, and gene disruption in Saccharomyces cerevisiae. Genes Dev 8, 1087-1105.

Calera, J. A., Zhao, X. J. \& Calderone, R. (2000). Defective hyphal development and avirulence caused by a deletion of the SSK1 response regulator gene in Candida albicans. Infect Immun 68, 518-525.

Cormack, B. P., Bertram, G., Egerton, M., Gow, N. A. R., Falkow, S. \& Brown, A. J. (1997). Yeast-enhanced green fluorescent protein (yEGFP): a reporter of gene expression in Candida albicans. Microbiology 143, 303-311.

Csank, C., Makris, C., Meloche, S., Schröppel, K., Röllinghoff, M., Dignard, D., Thomas, D. Y. \& Whiteway, M. (1997). Derepressed hyphal growth and reduced virulence in a VH1 family-related protein phosphatase mutant of the human pathogen Candida albicans. Mol Biol Cell 8, 2539-2551.

Csank, C., Schroppel, K., Leberer, E., Harcus, D., Mohamed, O., Meloche, S., Thomas, D. Y. \& Whiteway, M. (1998). Roles of the Candida albicans mitogen-activated protein kinase homolog, Cek1p, in hyphal development and systemic candidiasis. Infect Immun 66, 2713-2721.

Delbruck, S. \& Ernst, J. F. (1993). Morphogenesis-independent regulation of actin transcript levels in the pathogenic yeast Candida albicans. Mol Microbiol 10, 859-866.

Fonzi, W. A. \& Irwin, M. Y. (1993). Isogenic strain construction and gene mapping in Candida albicans. Genetics 134, 717-728.

Gale, C. A., Bendel, C. M., McClellan, M., Hauser, M., Becker, J. M., Berman, J. \& Hostetter, M. K. (1998). Linkage of adhesion, filamentous growth, and virulence in Candida albicans to a single gene, INT1. Science 279, 1355-1358.

Gietz, R. D., Schiestl, R. H., Willems, A. R. \& Woods, R. A. (1995). Studies on the transformation of intact yeast cells by the LiAc/ SS-DNA/PEG procedure. Yeast 11, 355-360.

Gilbert, W. \& Maxam, A. (1973). The nucleotide sequence of the lac operator. Proc Natl Acad Sci US A 70, 3581-3584.

Gimeno, C. J., Ljungdahl, P. O., Styles, C. A. \& Fink, G. R. (1992). Unipolar cell divisions in the yeast $S$. cerevisiae lead to filamentous growth: regulation by starvation and RAS. Cell 68, 1077-1090.

Guarente, L. (1983). Yeast promoters and lacZ fusions designed to study expression of cloned genes in yeast. Methods Enzymol 101, 181-191.

Guarente, L. \& Ptashne, M. (1981). Fusion of Escherichia coli lacZ to the cytochrome $c$ gene of Saccharomyces cerevisiae. Proc Natl Acad Sci US A 78, 2199-2203. 
Guthrie, C. \& Fink, G. R. (1991). Guide to Yeast Genetics and Molecular Biology. San Diego: Academic Press.

Hill, J., Donald, K. A., Griffiths, D. E. \& Donald, G. (1991). DMSOenhanced whole cell yeast transformation. [An erratum appears in Nucleic Acids Res 11, 6688.] Nucleic Acids Res 19, 5791.

Jacobson, R. H., Zhang, X. J., DuBose, R. F. \& Matthews, B. W. (1994). Three-dimensional structure of beta-galactosidase from E. coli. Nature 369, 761-766.

Kwon-Chung, K. B. (1992). Medical Mycology. Philadelphia: Lea \& Febinger.

Leberer, E., Harcus, D., Broadbent, I. D. \& 7 other authors (1996). Signal transduction through homologues of the Ste20p and Ste7p protein kinases can trigger hyphal formation in the pathogenic fungus Candida albicans. Proc Natl Acad Sci USA 93, 13217-13222.

Leberer, E., Ziegelbauer, K., Schmidt, A., Harcus, D., Dignard, D., Ash, J., Johnson, L. \& Thomas, D. Y. (1997). Virulence and hyphal formation of Candida albicans require the Ste20p-like protein kinase CaCla4p. Curr Biol 7, 539-546.

Leuker, C. E., Hahn, A. M. \& Ernst, J. F. (1992). $\beta$-Galactosidase of Kluyveromyces lactis (Lac4p) as reporter of gene expression in Candida albicans and C. tropicalis. Mol Gen Genet 235, 235-241.

Leuker, C. E., Sonneborn, A., Delbruck, S. \& Ernst, J. F. (1997). Sequence and promoter regulation of the PCK1 gene encoding phosphoenolpyruvate carboxykinase of the fungal pathogen Candida albicans. Gene 192, 235-240.

Liu, H., Kohler, J. \& Fink, G. R. (1994). Suppression of hyphal formation in Candida albicans by mutation of a STE12 homolog. Science 266, 1723-1726.

Lo, H. J., Kohler, J. R., DiDomenico, B., Loebenberg, D., Cacciapuoti, A. \& Fink, G. R. (1997). Nonfilamentous C. albicans mutants are avirulent. Cell 90, 939-949.

Myers, K. K., Sypherd, P. S. \& Fonzi, W. A. (1995). Use of $U R A 3$ as a reporter of gene expression in C. albicans. Curr Genet 27, 243-248.

Newport, G. \& Agabian, N. (1997). KEX2 influences Candida albicans proteinase secretion and hyphal formation. J Biol Chem 272, 28954-28961.

Odds, F. C. (1988). Candida and Candidosis, 2nd edn. London: Baillière Tindall.

Ohama, T., Suzuki, T., Mori, M., Osawa, S., Ueda, T., Watanabe, K. \& Nakase, T. (1993). Non-universal decoding of the leucine codon CUG in several Candida species. Nucleic Acids Res 21, 4039-4045.

Poch, O., L'Hote, H., Dallery, V., Debeaux, F., Fleer, R. \& Sodoyer, R. (1992). Sequence of the Kluyveromyces lactis beta-galacto- sidase: comparison with prokaryotic enzymes and secondary structure analysis. Gene 118, 55-63.

Rose, M., Casadaban, M. J. \& Botstein, D. (1981). Yeast genes fused to beta-galactosidase in Escherichia coli can be expressed normally in yeast. Proc Natl Acad Sci US A 78, 2460-2464.

Schroeder, C. J., Robert, C., Lenzen, G., McKay, L. L. \& Mercenier, A. (1991). Analysis of the lacZ sequences from two Streptococcus thermophilus strains: comparison with the Escherichia coli and Lactobacillus bulgaricus $\beta$-galactosidase sequences. $J$ Gen Microbiol 137, 369-380.

Sreekrishna, K. \& Dickson, R. C. (1985). Construction of strains of Saccharomyces cerevisiae that grow on lactose. Proc Natl Acad Sci U S A 82, 7909-7913.

Srikantha, T., Klapach, A., Lorenz, W. W., Tsai, L. K., Laughlin, L. A., Gorman, J. A. \& Soll, D. R. (1996). The sea pansy Renilla reniformis luciferase serves as a sensitive bioluminescent reporter for differential gene expression in Candida albicans. J Bacteriol 178, 121-129.

Srikantha, T., Tsai, L. K. \& Soll, D. R. (1997). The WHI1 gene of Candida albicans is regulated in two distinct developmental programs through the same transcription activation sequences. $J$ Bacteriol 179, 3837-3844.

Staab, J. F., Ferrer, C. A. \& Sundstrom, P. (1996). Developmental expression of a tandemly repeated, proline- and glutamine-rich amino acid motif on hyphal surfaces of Candida albicans. J Biol Chem 271, 6298-6305.

Stoldt, V. R., Sonneborn, A., Leuker, C. E. \& Ernst, J. F. (1997). Efg1p, an essential regulator of morphogenesis of the human pathogen Candida albicans, is a member of a conserved class of bHLH proteins regulating morphogenetic processes in fungi. EMBO J 16, 1982-1991.

Swoboda, R. K., Bertram, G., Delbruck, S., Ernst, J. F., Gow, N. A. R., Gooday, G. W. \& Brown, A. J. (1994). Fluctuations in glycolytic mRNA levels during morphogenesis in Candida albicans reflect underlying changes in growth and are not a response to cellular dimorphism. Mol Microbiol 13, 663-672.

Timpel, C., Strahl-Bolsinger, S., Ziegelbauer, K. \& Ernst, J. F. (1998). Multiple functions of Pmt1p-mediated protein O-mannosylation in the fungal pathogen Candida albicans. J Biol Chem 273, 20837-20846.

Wirsching, S., Michel, S., Kohler, G. \& Morschhauser, J. (2000). Activation of the multiple drug resistance gene MDR1 in fluconazole-resistant, clinical Candida albicans strains is caused by mutations in a trans-regulatory factor. J Bacteriol 182, 400-404.

Received 12 December 2000; accepted 15 January 2001. 